\title{
Ultrafast X-ray Diffraction of Laser-irradiated Crystals
}

\author{
P.A. Heimann, ${ }^{1}$ J. Larsson, ${ }^{2}$ Z. Chang, ${ }^{3}$ A. Lindenberg, ${ }^{2}$ P.J. Schuck, ${ }^{2}$ E. Judd, ${ }^{2}$ \\ H.A. Padmore, ${ }^{1}$ P.H. Bucksbaum, ${ }^{3}$ R.W. Lee, ${ }^{4}$ M. Murnane, ${ }^{3}$ H. Kapteyn, ${ }^{3}$ J.S. Wark ${ }^{5}$ \\ and R.W. Falcone ${ }^{2}$
}

'Advanced Light Source, Accelerator and Fusion Research Division, Lawrence Berkeley National Laboratory, Berkeley, CA $94720,{ }^{2}$ Physics Department, University of California, Berkeley, CA $94720,{ }^{3}$ Center for Ultrafast Optical Science, University of Michigan, Ann Arbor, MI 48109, ${ }^{4}$ Lawrence Livermore National Laboratory, Livermore, CA 94551, ${ }^{5}$ Department of Physics, Clarendon Laboratory, University of Oxford, Oxford OX1 3PU, U.K.

\begin{abstract}
An apparatus has been developed for measuring time-dependent $\mathrm{x}$-ray diffraction. X-ray pulses from an Advanced Light Source bend magnet are diffracted by a sagittally-focusing Si (111) crystal and then by a sample crystal, presently InSb (111). Laser pulses with $100 \mathrm{fs}$ duration and a repetition rate of $1 \mathrm{KHz}$ irradiate the sample inducing a phase transition. Two types of detectors are being employed: an $\mathrm{x}$-ray streak camera and an avalanche photodiode. The streak camera is driven by a photoconductive switch and has a 2 ps temporal resolution determined by trigger jitter. The avalanche photodiode has high quantum efficiency and sufficient time resolution to detect single x-ray pulses in ALS two bunch or 'camshaft' operation. A beamline is under construction dedicated for time resolved and micro-diffraction experiments. In the new beamline a toroidal mirror collects 3 mrad horizontally and makes a 1:1 image of the bend magnet source in the $\mathrm{x}$-ray hutch. A laser induced phase transition has been observed in InSb occuring within 70 ps.
\end{abstract}

\section{INTRODUCTION}

Laser sources currently produce ultrashort ( $<1 \mathrm{ps)} \mathrm{pulses} \mathrm{at} \mathrm{infra-red,} \mathrm{visible,} \mathrm{and} \mathrm{ultraviolet} \mathrm{wavelengths} \mathrm{which} \mathrm{can}$ be used to study the dynamics of valence electrons in atoms, molecules, and solids. Studies of laser-induced phase transitions have involved time-resolved reflectivity and second-harmonic generation of optical light pulses. However, to directly probe structural properties of molecules and solids it is advantageous to use $x$-ray radiation. For example, conformational changes were recently studied in the ns time domain at the European Synchrotron Radiation Facility, using Laue diffraction from a rapidly evolving myoglobin sample. (1) Laue diffraction images were obtained from a single pulse of $\mathrm{x}$-rays from the storage ring. With a resolution in the range of $1 \mathrm{ps,} x$-ray diffraction techniques could be applied to the study of ultrafast processes such as laser-induced phase transitions and transient excited modes in solids.

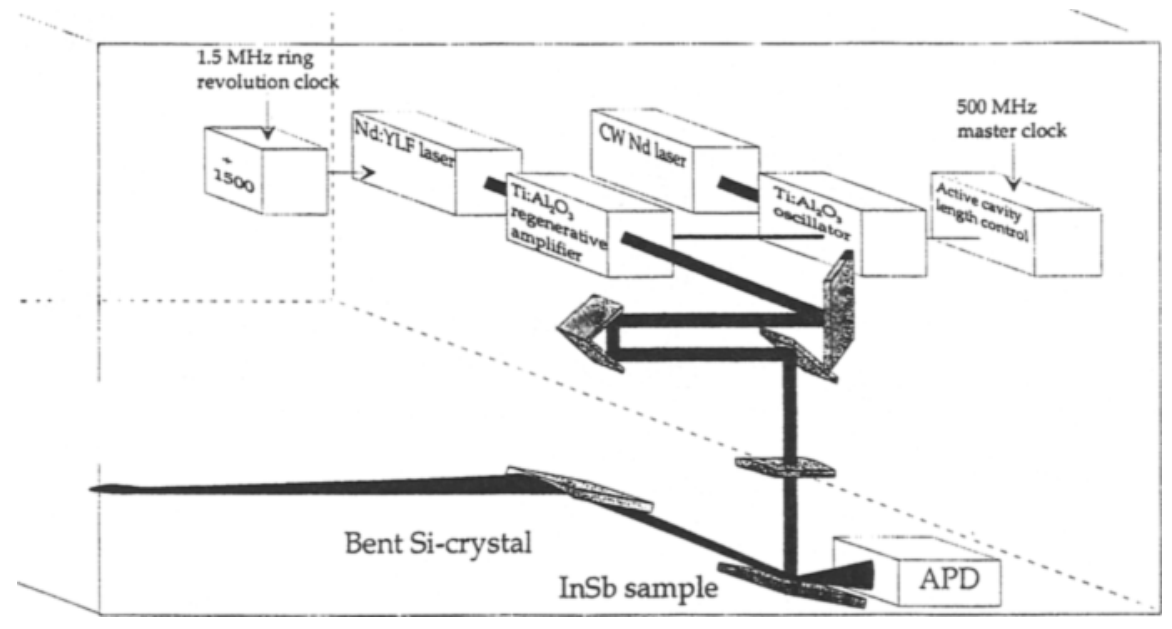

FIGURE 1. Experimental set-up showing the x-ray radiation from the synchrotron, two crystals, laser system, and avalanche photodiode detector. 
We have established an $\mathrm{x}$-ray diffraction experiment at the Advanced Light Source (ALS) synchrotron, which will enable the study of ultrafast structural dynamics. These dynamics are initiated by $100 \mathrm{fs}$ laser pulses from a $\mathrm{Ti}_{2} \mathrm{Al}_{2} \mathrm{O}_{3}$ laser system, which is synchronized to the electron bunches in the storage ring. Radiation from a bend magnet beamline diffracts from two crystals into either an $\mathrm{x}$-ray streak camera or an avalanche photodiode. This apparatus has enabled us to conduct pump-probe x-ray diffraction experiments in which we irradiate an InSb crystal with a $100 \mathrm{fs}$ laser pulse, and probe the interaction with a $70 \mathrm{ps} x$-ray pulse from the ALS. The different techniques are described in the next section. The results of measurements demonstrating the capability of the instrumentation are presented in section 3 .

\section{TECHNIQUES}

The experimental apparatus, including synchrotron radiation beamline, crystal chamber, detectors and laser system is shown in Fig. 1. The initial form of this apparatus has been described previously in Ref. 2. The ALS storage ring is operated with different timing modes. Some experiments were performed with the ALS in a double-bunch mode, for which the maximum beam current is $40 \mathrm{~mA}$ and the pulse repetition rate is $3 \mathrm{MHz}$. Other measurements were conducted during 'camshaft' operation, in which an isolated bucket, having a $66 \mathrm{~ns}$ gap before and a $16 \mathrm{~ns}$ gap afterwards, is filled to $20 \mathrm{~mA}$. The lifetime of this isolated electron bunch is however shorter than the lifetime in double-bunch mode. The duration of $\mathrm{x}$-ray pulses from the ring varies with the current in the electron bunches, $\Delta t \sim \mathrm{i}^{-1 / 3}$, but is typically $30-80 \mathrm{ps}$. (3)

To date the beamline 10.3.2 has been used for these experiments. This beamline delivers the unfocused, white radiation through a Be window into a $\mathrm{x}$-ray hutch. The available photon energy range is from about $3 \mathrm{keV}$ where the $\mathrm{Be}$ window transmission cuts off to about $12 \mathrm{keV}$ where the bend magnet spectrum decreases at $1.9 \mathrm{GeV}$ electron beam energy. The angular acceptance of the $\mathrm{x}$-ray radiation is determined by apertures in the endstation, typically 0.3 mrad horizontally by $0.03 \mathrm{mrad}$ vertically. These apertures are set to provide a $1 \mathrm{~mm}^{2}$ footprint on the sample crystal not larger than the footprint of the laser beam.

The ALS beamline 7.3.3 is under construction and is specifically designed for time resolved $\mathrm{x}$-ray diffraction and micro-diffraction experiments. Figure 2 shows a layout of this beamline. A toroidal mirror provides a 1:1 double focus of the bend magnet source in the endstation hutch. The angular acceptance of the mirror, $3 \mathrm{mrad}$ horizontally $\mathrm{x} 0.2 \mathrm{mrad}$ vertically, results in a 40-fold increase in the useable flux for time-resolved diffraction experiments compared with the other beamline without focusing. The energy range extends from about $2 \mathrm{keV}$ determined by the thickness of the $\mathrm{C}$ filter and $\mathrm{Be}$ window to about $12 \mathrm{keV}$ resulting from both the bend magnet spectrum and the mirror reflectivity.

The toroidal mirror produces a 1:1 image of the ALS electron beam in a center bend magnet, whose dimensions are $250 \mu \mathrm{m}$ horizontally $\times 50 \mu \mathrm{m}$ vertically $(\mathrm{fwhm})$. The mirror has a grazing incidence angle of $5.4 \mathrm{mrad}$ and equal source and image distances of $16 \mathrm{~m}$. Consequently, the sagittal radius is polished to be $87 \mathrm{~mm}$, while the tangential radius is bent to $2.94 \mathrm{~km}$. The mirror substrate has dimensions of $700 \times 75 \times 75 \mathrm{~mm}^{3}$ and is manufactured from single crystal silicon. The mirror is bent with equal couples applied by a leaf spring bender. The bender mechanism is attached to the mirror through metal blocks glued onto the ends of the mirror. The mirror, which absorbs $5 \mathrm{~W}$, is not actively cooled. Analysis of the thermal and gravitational deformations of the mirror shows that these errors can be compensated by adjusting the bending of the mirror to a residual error of $\sim 1 \mu \mathrm{rad}$ rms. (4)

Some additional components of beamline 7.3.3 are shown in figure 2. A $10 \mu \mathrm{m}$ thick $\mathrm{C}$ filter reduces the power absorbed in the Be window. Horizontal and vertical apertures downstream of the mirror chamber adjust the angular acceptance

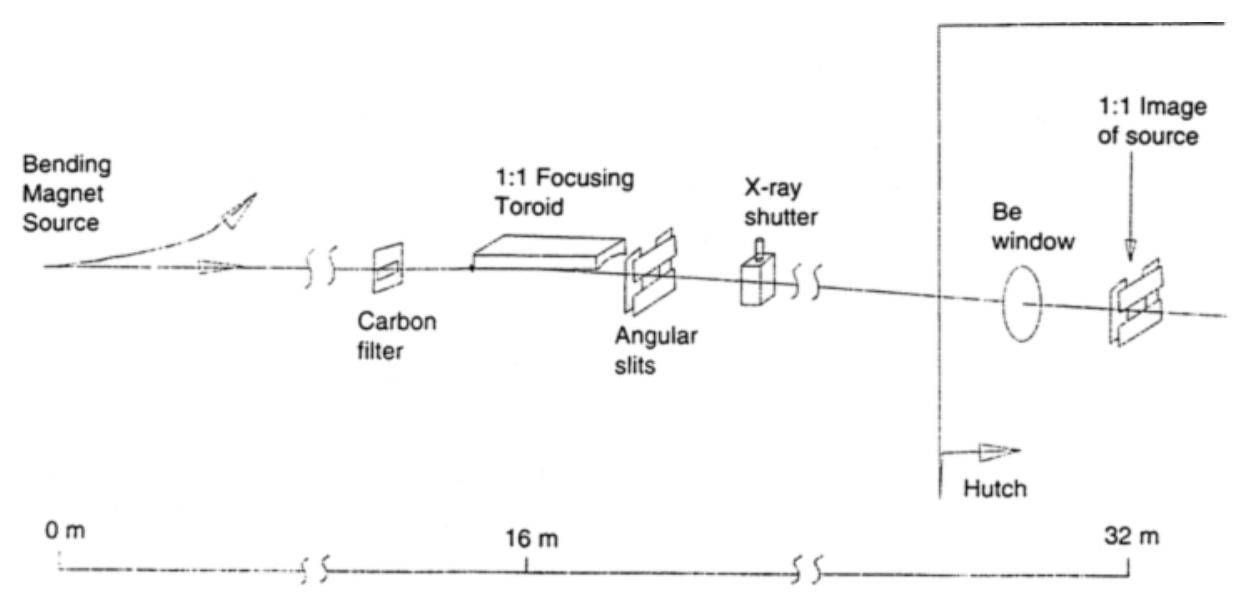

FIGURE 2. Layout of ALS beamline 7.3.3. 
for the x-ray micro-diffraction optics, which have a limited collection aperture. A fixed bremsstrahlung backstop blocks the high energy radiation. In addition, there is an $\mathrm{x}$-ray shutter, which allows personnel to enter the hutch. The Be window, 50 $\mu \mathrm{m}$ thick and spherical in shape, separates the UHV of the beamline from the inferior vacuum or $\mathrm{He}$ atmosphere of the experiments. A hutch contains the endstations, while an adjoining room encloses the laser system.

In the present sychrotron beamline 10.3.2, the white bend magnet radiation is monochromatized by a bent $\mathrm{Si}$ (111) crystal, and a sample crystal is illuminated by the laser. The crystals diffract in the vertical plane, corresponding to spolarization. The Bragg angle of the $\mathrm{Si}$ (111) crystal is fixed at $22.5^{\circ}$ which results in the reflection of $5 \mathrm{keV} \mathrm{x}$-rays. In order to increase the $\mathrm{x}$-ray intensity within the laser illuminated area on the sample crystal, the first crystal is sagittally curved. The thin Si crystal is bent into a cylinder with $\mathrm{R} \sim 0.75 \mathrm{~m}$ corresponding to a focal distance of $1 \mathrm{~m}$. Two rectangular springs bend the rectangular Si crystal, which has a length / width ratio $\sim 4$ in order to reduce anticlastic bending. (5) The crystal bender is actuated by a motor driven slide allowing the line focus to be positioned either at the sample or the detector. The width of the focal line was measured with the streak camera to be about $100 \mu \mathrm{m}$.

In the experiment described below, the sample is InSb (111) chosen to have comparable penetration of laser and $\mathrm{x}$-ray radiation into the crystal. At high laser fluence the pump-laser penetration depth is in general limited by free-carrier absorption to about $100 \mathrm{~nm}$. Because InSb has L-shell binding energies between 3.7 and $4.7 \mathrm{keV}$, the penetration of $5 \mathrm{keV} \mathrm{x}$ rays is reduced by photoabsorption. An alternative approach is to use thin crystals grown on substrates.

The laser system consists of a Ti: $\mathrm{Al}_{2} \mathrm{O}_{3}$ laser oscillator pumped by a frequency-doubled Nd-laser (Spectra Physics: Millenia), and a Ti: $\mathrm{Al}_{2} \mathrm{O}_{3}$ regenerative amplifier system (Positive Light: Spitfire/Merlin) operating at a repetition rate of $1 \mathrm{kHz}$. The pulse duration is approximately $100 \mathrm{fs}$ and the pulse energy is about $1 \mathrm{~mJ}$. Synchronization of the laser pulses with the synchrotron pulses is achieved by locking the laser-oscillator repetition frequency (set by the oscillator cavity length) to the master RF clock of the ALS. (6) From the error signal observed in the stabilization electronics, we estimate the jitter between the laser and the synchrotron pulses to be about $5 \mathrm{ps}$. The delay between the laser and the $\mathrm{x}$-ray pulse can be varied using an optical delay line in the laser beam path. The laser beam is focused on the InSb (111) sample at a fluence of about $30 \mathrm{~mJ} / \mathrm{cm}^{2}$, below the threshold for rapid and visible damage.

Two types of detectors have been employed for time resolved $\mathrm{x}$-ray diffraction: $\mathrm{x}$-ray streak cameras and avalanche photodiodes. Using a Si photodiode 1500 diffracted photons / pulse were collected. Because of the low x-ray flux / shot and the $\mathrm{kHz}$ repetition rate of the laser, nearly all measurements have been done by averaging over multiple pulses. Multiple shot data acquisition is possible because at the fluence used in these studies irreversable damage of the InSb is not observed even after exposure times as long as 15 minutes.

The $\mathrm{x}$-ray streak camera has been described in Ref. 7 and 8 . Briefly, $\mathrm{x}$-rays strike a CsI photocathode generating electrons, which are accelerated through a slit and deflected by sweep plates. The electrons are then focused with a magnetic lens onto an amplifying pair of microchannel plates and a phosphor screen. This $\mathrm{x}$-ray streak camera has the capability to operate at a high repetition rate $(1 \mathrm{kHz}$ ), high time resolution (540 $\mathrm{fs}$ in single shot mode), and low jitter triggering using a photoconductive switch. On the other hand, streak cameras have a low x-ray quantum efficiency, about $1 \%$, because of the $100 \mathrm{~nm}$ thick CsI photocathode.

Jitter in the streak camera timing with respect to the laser pulse was measured using a test setup. About $20 \mathrm{~mJ}$ of $800 \mathrm{~nm}$ light from the $\mathrm{Ti}: \mathrm{Al}_{2} \mathrm{O}_{3}$ laser was used to trigger a $\mathrm{GaAs}$ photoconductive switch, (9) which drives the high-voltage pulse to the sweep plates of the streak camera. UV light, with a wavelength of $266 \mathrm{~nm}$, was obtained by frequency mixing the $800 \mathrm{~nm}$ light in two KDP crystals. The UV light was split into two pulses separated by $20 \mathrm{ps,} \mathrm{using} \mathrm{a} \mathrm{fused} \mathrm{silica}$ window of known thickness inserted halfway into the beam. This UV double-pulse was then incident on the photocathode and used to calibrate the time response and relative jitter of the streak camera. The resulting effective temporal resolution of the streak camera system was $2 \mathrm{ps}$ in this averaging mode (here about 5000 pulses were summed).

Alternatively, the diffracted $x$-rays are detected by an avalanche photodiode (Advanced Photonix, Inc.). This detector with an active area of $1 \mathrm{~cm}^{2}$ has near unity quantum efficiency and a gain of 200 . (10) The response time is $10 \mathrm{~ns}$, sufficient to resolve individual ALS $x$-ray pulses in either two bunch or 'camshaft' mode. The signal is recorded using a digital oscilloscope or a gated-integrator connected to a computer. In all the pump probe experiments, the gated-integrator was used, since that allowed us to record data at $1 \mathrm{KHz}$, as determined by the laser repetition rate. When using the avalanche photodiode, the time resolution is determined by the ALS x-ray pulse duration, about $70 \mathrm{ps}$.

\section{MEASUREMENTS}

As a test of our ability to observe time-resolved events, we measured the duration of the diffracted ALS pulses. For this test the sample crystal was $\mathrm{Si}(111)$ and no laser irradiation was applied. The $\mathrm{x}$-ray streak camera was used as the detector. The result of the measurement is shown in Fig. 3. Data was obtained by averaging over 2000 pulses. The observed pulse duration of $70 \mathrm{ps}$ is in good agreement with previous measurements of the ALS pulse duration. (3) This measurement confirms that the laser and $x$-ray streak camera are synchronized to the ALS time structure to within a fraction of 70 ps. 


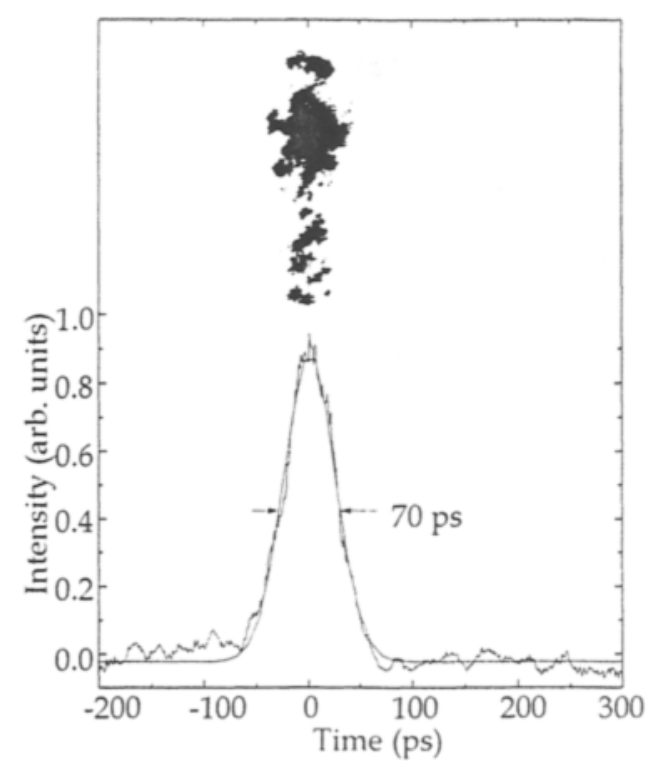

FIGURE 3. The pulse duration of the ALS was measured to be $70 \mathrm{ps}$ with a streak camera operating in an averaging mode. The sample was $\mathrm{Si}(111)$. Both the camera output and a lineout of this data are shown.

In the following measurement a pump-probe technique is employed. In Fig. 4 the diffracted x-ray intensity is shown as a function of delay between the heating pulse and the $x$-ray probe. The length of the laser beam path is varied using the optical delay line. The detector was an avalanche photodiode. The drop in diffraction intensity has a time-resolution limited by the ALS-pulse duration. By differentiating the signal, the ALS pulse shape is retrieved as shown as the dashed trace in Fig. 4. About 10 ps broadening is attributed to timing jitter and the relatively course time steps were used. The data in Fig. 4 comprises about $10^{5}$ laser pulses at the repetition rate of $1 \mathrm{KHz}$.

The drop in diffracted intensity is interpreted as resulting from the melting of a layer at the surface of the InSb crystal. The melted, disordered layer absorbs a fraction of the $\mathrm{x}$-ray intensity. Before the next laser shot, the crystal lattice has regrown, and the crystal has equilibrated at an elevated temperature, in this case $50{ }^{\circ} \mathrm{C}$ above ambient temperature. It is concluded that a phase transition in InSb occurs with a characteristic time less than the ALS pulse duration, i.e. $<70$ ps.

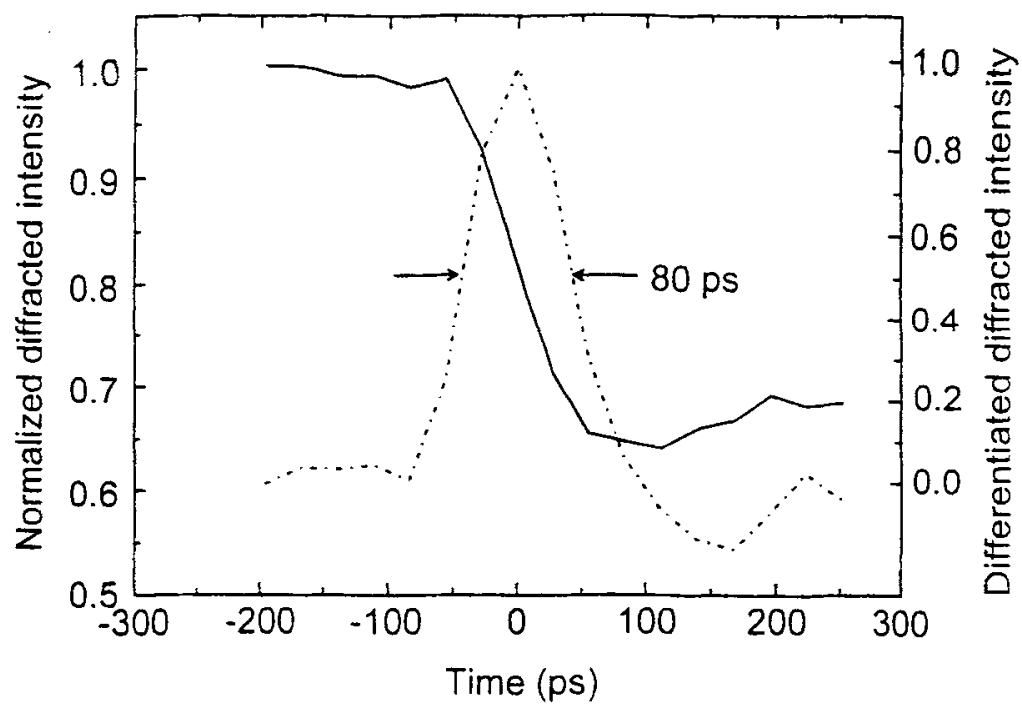

FIGURE 4. X-ray diffraction efficiency as a function of delay between the heating laser pulse and the $x$-ray pulse (solid line) The sample was InSb(111). The differential of the x-ray diffraction efficiency yields the expected pulse shape for the ALS pulse (dashed line). 
In conclusion, we have demonstrated an apparatus combing synchrotron radiation with an ultrafast laser system, a crystal diffractometer and time resolved detectors. A beamline with focusing toroidal mirror is under construction for these experiments. The $\mathrm{x}$-ray streak camera has temporal resolution of $2 \mathrm{ps}$ operating in an averaging mode, for events that are triggered by ultrafast laser pulses. Our set-up will allow us to pursue laser-pump / $\mathrm{x}$-ray-probe experiments to monitor structural changes in materials with ultrafast time resolution. In a first set of experiments, we show evidence of a laserinduced phase transition in InSb, where disorder is induced on a timescale $<70 \mathrm{ps}$.

\section{ACKNOWLEDGMENTS}

The contributions of Alistair MacDowell, Keith Franck, Robert Patton, Neal Hartman, Andrew Grieshop, Robert Duarte and Ted Lauritzen to the design and construction of ALS beamline 7.3.3 are acknowledged. This work was supported by an Academic Research Infrastructure Instrumentation Grant from the National Science Foundation, and the Department of Energy through Lawrence Berkeley National Laboratory and Lawrence Livermore National Laboratory.

\section{REFERENCES}

1. Bourgeois, D., Ursby, T., Wulff, M., Pradervand, C., Legrand, A., Schildkamp, W., Laboure, S., Srajer, V., Teng, T. Y., Roth, M., and Moffat, K., J. Synchrotron Rad. 3, 65-74 (1996); Sanjer, V., Teng, T. Y., Ursby, T., Pradervand, C., Ren, Z., Adachi, S. I., Schildkamp, W., Bourgeois, D., Wulff, M., and Moffat, K., Science 274, 1726-1729 (1996).

2. Larsson, J., Chang, Z., Judd, E., Schuck, P. J., Falcone, R. W., Heimann, P. A., Padmore, H. A., Kapteyn, H. C., Bucksbaum, P.H., Murnane, M. M., Lee, R. W., Machacek, A., Wark, J. S., Liu, X., and Shan, B., Optics Lett. 22, 1012-1014 (1997).

3. Keller, R., Renner, T., and Massoletti, D. J., AIP Conference Proceedings 390, 240-247 (1996).

4. Hartman, N., and Grieshop, A., LSME 712.

5. Ferrer, S., Krisch, M., de Bergevin, F. and Zontone, F., Nucl. Instrum. \& Methods A 311, 444-447 (1992).

6. Schoenlein, R.W., Leemans, W.P., Chin, A.H., Volfbeyn, P., Glover, T.E., Balling, P., Zolotorev, M., Kim, K.-J., Chattopdhyay, S., and Shank, C.V., Science 274, 236-238 (1996).

7. Chang, Z., Rundquist, A., Zhou, J., Murnane, M. M., Kapteyn, H. C., Liu, X., Shan, B., Liu, J., Niu, L., Gong, M., and Zhang, X., Appl. Phys. Lett. 69, 133-135 (1996).

8. Chang, Z., Rundquist, A., Wang, H., Kapteyn, H. C., Murnane, M. M., Liu, X., Shan, B., Liu, J., Niu, L., Gong, M., Zhang, X., and Lee, R., SPIE 2869, to be published (1996).

9. Knox, W., and Mourou, G., Appl. Phys. Lett. 36, 623-626 (1980); Yen, R., Downey, M. M., Shank, C.V., and Auston, D.H, Appl. Phys. Lett. 44, 718-720 (1984); Maksimchuk, A., Kim, M., Workman, J., Korn, G., Squier, J., Du, D., Umstadter, D., and Bouvier, M., Rev. Sci. Instrum. 67, 697-699 (1996).

10. Gullikson, E.M., Gramsch, E., and Szawlowski, M., Applied Optics 34, 4662-4668 (1995). 